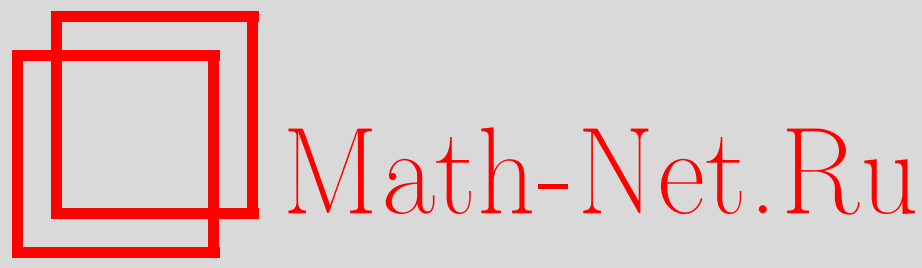

В. А. Копытцев, В. Г. Михайлов, Оценки для распределения минимального расстояния случайного линейного кода, Дискрет. матем., 2015, том 27, выпуск 2, 45-55

DOI: https://doi.org/10.4213/dm1324

Использование Общероссийского математического портала Math-Net.Ru подразумевает, что вы прочитали и согласны с пользовательским соглашением http://www . mathnet.ru/rus/agreement

Параметры загрузки:

IP : 52.6 .47 .48

26 апреля 2023 г., 15:00:44

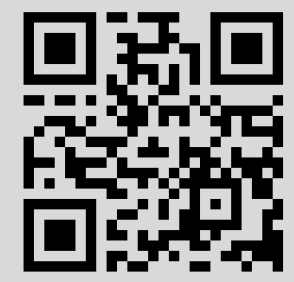




\title{
Оценки для распределения минимального расстояния случайного линейного кода
}

\author{
() 2015 г. В. А. Копытцев*, В. Г. Михайлов**
}

Исследуется функция распределения минимального расстояния (минимального веса ненулевых векторов) случайного линейного кода над конечным полем. Получены явные оценки в форме неравенств и асимптотические оценки для этой функции распределения.

Ключевые слова: минимальное расстояние линейного кода, система случайных включений над конечным полем, минимальный вес вектора в множестве решений, оценка точности пуассоновской аппроксимации.

\section{1. Введение}

Пусть $n>m$ - натуральные числа, а $\mu(n, m)$ - минимальное расстояние (минимальный вес ненулевых векторов) случайного линейного $(n, m)$-кода над конечным полем $K=G F(q)$. Под линейным $(n, m)$-кодом $K_{n, m}$ понимается множество всех векторов некоторого $m$-мерного подпространства в $n$-мерном пространстве $K^{n}$. Под весом $w(x)$ вектора $x \in K^{n}$ понимается число ненулевых элементов в записи $x=\left(x_{1}, \ldots, x_{n}\right)$. Подробнее об этих и других понятиях теории кодирования см. [1]-[3].

Мы рассматриваем случай, когда подпространство $K_{n, m}$ выбирается в соответствии с равномерным распределением на множестве всех $m$-мерных подпространств в пространстве $K^{n}$. Тогда $\mu(n, m)$ представляет собой случайную величину со значениями в множестве натуральных чисел, распределение которой зависит от параметров $n$ и $m$.

Наша основные цели - получить явные оценки в форме неравенств для функции распределения величины $\mu(n, m)$, определить диапазон, в котором сосредоточена основная масса распределения величины $\mu(n, m)$, а также получить выражения для главных членов и оценки порядка остаточных членов в асимптотических формулах для этого распределения. В доказательствах используются полученные недавно авторами явные оценки точности пуассоновской аппроксимации для распределения числа решений случайных включений и уравнений над конечным полем.

*Место работы: Академия криптографии Российской Федерации, e-mail: kopytcev2012@mail.ru

** Место работы: Математический институт им. В.А.Стеклова РАН, e-mail: mikhail@mi.ras.ru 


\section{2. Формулировки результатов}

Так же, как в [4] и [5], определим числовую функцию $\rho(D)$ множества $D \subset K^{n}$ следующим образом. Обозначим через $N\left(k_{1}, k_{2}, k_{3}, d, D\right)$ число решений уравнения

$$
k_{1} u^{1}+k_{2} u^{2}+k_{3} u^{3}=d
$$

относительно тройки векторов $\left(u^{1}, u^{2}, u^{3}\right) \in D^{3}$, где $k_{1}, k_{2}, k_{3} \in K \backslash\{0\}, d \in K^{n}$. Пусть

$$
N(D)=\max _{k_{1}, k_{2}, k_{3}, d} N\left(k_{1}, k_{2}, k_{3}, d, D\right) \text {. }
$$

Тогда

$$
\rho(D)=N(D) /|D|^{2}
$$

Известно (см. [4]), что

$$
|D|^{-1} \leqslant \rho(D) \leqslant 1 \text {. }
$$

Далее в роли множества $D$ выступает множество $D_{r}^{*}$, которое определяется следующим образом. Пусть

$$
D_{r}=\left\{x \in K^{n}: 1 \leqslant \omega(x) \leqslant r\right\} .
$$

Разобьем множество (3) на классы подобных элементов (элементы $x^{\prime}, x^{\prime \prime} \in K^{n}$ назовем подобными, если $x^{\prime}=k x^{\prime \prime}, k \in K \backslash\{0\}$ ). Выберем по одному произвольному элементу из каждого класса. Множество $D_{r}^{*}$ составим из выбранных элементов.

Отметим, что

$$
\left|D_{r}^{*}\right|=\frac{\left|D_{r}\right|}{q-1}=\sum_{i=1}^{r} C_{n}^{i}(q-1)^{i-1}
$$

и согласно определению функции $\rho(D)$

$$
\rho\left(D_{r}^{*}\right) \leqslant(q-1)^{2} \rho\left(D_{r}\right) .
$$

Положим

$$
\lambda_{r}(n, m)=\frac{\left|D_{r}^{*}\right|}{q^{n-m}}=\frac{1}{q^{n-m}} \sum_{i=1}^{r} C_{n}^{i}(q-1)^{i-1} .
$$

Теорема 1. Выполнено неравенство

$$
\begin{gathered}
\left|\mathbf{P}\{\mu(n, m)>r\}-e^{-\lambda_{r}(n, m)}\right| \leqslant \\
\leqslant \rho\left(D_{r}^{*}\right) \cdot\left[A\left(\lambda_{r}(n, m),\left|D_{r}^{*}\right|\right)+B\left(\lambda_{r}(n, m)\right)\right]+q^{m-n} e^{\lambda_{r}(n, m)}+q^{-m},
\end{gathered}
$$

где

$$
\begin{gathered}
A\left(\lambda,\left|D_{r}^{*}\right|\right)=\lambda^{2} e^{q \lambda-1+\left|D_{r}^{*}\right|^{-1}}, \\
B(\lambda)=\frac{1}{\lambda-1}\left[\left(e^{\lambda}-1-\lambda-\frac{\lambda^{2}}{2}\right)-\lambda^{2}\left(e-\frac{5}{2}\right)\right], \quad \lambda \neq 1,
\end{gathered}
$$

$u B(1)=3-e$.

Приведем асимптотический вариант этого утверждения

Следствие 1. Пусть $n \rightarrow \infty, 0<\delta \leqslant m / n \leqslant \delta^{\prime}<1 u \lambda_{r}(n, m)=O(1)$. Тогда

$$
\left.\mathbf{P}\{\mu(n, m)>r\}=e^{-\lambda_{r}(n, m)}+O\left(\rho\left(D_{r}\right) \cdot \lambda_{r}^{2}(n, m)\right)\right) .
$$


Из (7) и (10) вытекает необходимость исследования величин $\rho\left(D_{r}^{*}\right)$ и $\rho\left(D_{r}\right) . \mathrm{B}$ следующей теореме предлагается оценка скорости убывания величины $\rho\left(D_{r}\right)$ при переходе к пределу по $r, n \rightarrow \infty$.

Теорема 2. Пусть $r \rightarrow \infty$ так, что при некотором $0<\beta<1$

$$
\frac{r}{n} \leqslant(1-\beta) R(q), \quad R(q)=\frac{q-1}{q}\left(1-\sqrt{1-\frac{q}{2(q-1)}}\right) .
$$

Тогда при любом $0<\varepsilon<1$

$$
\rho\left(D_{r}\right)=O\left(r^{\varepsilon-1}\right) .
$$

Замечание 1. В работах [6] и [7] были получены следующие неравенства для функции распределения минимального веса ненулевых векторов случайного линейного $(n, m)$-кода (в [6] - для двоичного случая, а в [7] для случая простого поля $G F(p))$ :

$$
\begin{gathered}
\mathbf{P}\{\mu(n, m) \leqslant r\} \geqslant\left(1+\frac{\left(p^{n}-p^{m}\right)\left(1-p^{-n}\right)}{\left(p^{n}-p\right)\left(1-p^{-m}\right)} \frac{p-1}{\lambda_{r}(n, m)}\right)^{-1}, \\
\mathbf{P}\{\mu(n, m) \leqslant r\} \leqslant \frac{1-p^{-m}}{1-p^{-n}} \lambda_{r}(n, m) .
\end{gathered}
$$

Применив оценку теоремы 2 в (7) и (10), нетрудно убедиться, что при переходе к пределу по $n, m, n-m \rightarrow \infty$, оценки (7) и (10) точнее оценок (13) и (14). Однако явных выражений или оценок для $\rho\left(D_{r}\right)$ пока не имеется.

Исследуем теперь поведение функции распределения случайной величины $\mu(n, m)$ при согласованном росте всех трех параметров $n, m, r$.

Рассмотрим при $q, n \in\{2,3, \ldots\}, x \in(0,1)$ функцию

$$
M_{q, n}(x)=-x \ln x-(1-x) \ln (1-x)+x \ln (q-1)-\frac{1}{n} \ln \sqrt{2 \pi n x(1-x)} .
$$

Пусть число $\kappa=\kappa(n, m)$ является решением уравнения

$$
M_{q, n}(x)=\left(1-\frac{m}{n}\right) \ln q,
$$

a

$$
q_{s}(n, m)=\frac{1-\kappa}{(q-1)(1-\kappa)-\kappa}\left((q-1) \frac{1-\kappa}{\kappa}\right)^{s-\{n \kappa\}}, \quad s=0, \pm 1, \ldots,
$$

где $\{a\}=a-\lfloor a\rfloor,\lfloor a\rfloor-$ дробная и целая части числа $a$.

Замечание 2. Нетрудно показать, что производная функции $M_{q, n}(x)$ положительна на интервале $(0,1 / 2)$. Поэтому при любых $m, n$, удовлетворяющих условию

$$
0<\left(1-\frac{m}{n}\right) \ln q<M_{q, n}\left(\frac{1}{2}\right)=\ln 2+\frac{1}{2} \ln (q-1)-\frac{1}{n} \ln \sqrt{\frac{\pi n}{2}},
$$

уравнение (15) имеет единственное решение на интервале $(0,1 / 2)$.

В следующей теореме параметр $\kappa=\kappa(n, m)$ связывает величину $r$ с величинами $n, m$.

Теорема 3. Пусть $n, m \rightarrow \infty, r=\lfloor\kappa(n, m) n\rfloor+s, s^{2}=o(n)$, выполнено условие

$$
0<c_{1} \leqslant \kappa(n, m) \leqslant c_{2}<R_{1}(q)=\min \left\{\frac{q-1}{q-1+e}, R(q)\right\} .
$$


1) Если $s=O(1)$, то при любом $0<\varepsilon<1$

$$
\mathbf{P}\{\mu(n, m)-\lfloor n \kappa(n, m)\rfloor>s\}=e^{-q_{s}(n, m)}+O\left(n^{\varepsilon-1}\right) .
$$

2) Если $s \rightarrow-\infty$, то при любом $0<\varepsilon<1$

$$
\begin{gathered}
\mathbf{P}\{\mu(n, m)-\lfloor n \kappa(n, m)\rfloor>s\}=e^{-q_{s}(n, m)}+ \\
+O\left(\frac{s^{2}+\ln n}{n}+\frac{q_{s}^{2}(n, m)}{n^{1-\varepsilon}}\right) .
\end{gathered}
$$

Замечание 3. Из определений следует, что $R_{1}(2)=1 /(1+e)$, а $1 / 3=R_{1}(3)=$ $R(3)>R_{1}(4)=R(4)>\ldots>1-2^{-1 / 2}$.

Замечание 4. Из условия (17) следует, что $(q-1)(1-\kappa) / \kappa>e$, а величина $q_{s}(n, m)$ в выражении (18) растет вместе с $s$.

\section{3. Доказательство теоремы 1}

Пусть $A_{n-m, n}$ случайная матрица размера $(n-m) \times n$, состоящая из независимых в совокупности равномерно распределенных на $K=G F(q)$ случайных элементов.

Пусть $\xi_{r}(n, n-m)$ и $\xi_{r}^{*}(n, n-m)$ - числа решений системы $A_{n-m, n} x=0$, удовлетворяющих условиям $x \in D_{r}$ и $x \in D_{r}^{*}$ соответственно. Нам понадобится следующая оценка точности пуассоновской аппроксимации для $\xi_{r}^{*}(n, n-m)$. Она вытекает непосредственно из следствия 1 работы [5].

Лемма 1. Выполнено неравенство

$$
\begin{gathered}
\left|\mathbf{P}\left\{\xi_{r}^{*}(n, n-m)=0\right\}-e^{-\lambda_{r}(n, m)}\right| \leqslant \\
\leqslant \rho\left(D_{r}^{*}\right) \cdot\left[A\left(\lambda_{r}(n, m),\left|D_{r}^{*}\right|\right)+B\left(\lambda_{r}(n, m)\right)\right]+q^{m-n} e^{\lambda_{r}(n, m)},
\end{gathered}
$$

где все величины из правой части определены в теореме 1.

Пусть $\mu\left(x: A_{T, n} x=0\right)$ - минимальный вес ненулевого вектора в множестве решений системы $A_{T, n} x=0$ относительно вектора неизвестных $x \in K^{n}$. Согласно нашим определениям

$$
\left\{\mu\left(x: A_{n-m, n} x=0\right)>r\right\}=\left\{\xi_{r}(n, n-m)=0\right\}=\left\{\xi_{r}^{*}(n, n-m)=0\right\} .
$$

Заметим, что из независимости и равномерности распределения элементов матрицы $A_{n-m, n}$ следует, что

$$
\mathbf{P}\left\{\mu\left(x: A_{n-m, n} x=0\right)>r \mid \operatorname{rank}\left(A_{n-m, n}\right)=n-m\right\}=\mathbf{P}\{\mu(n, m)>r\} .
$$

Поэтому

$$
\begin{gathered}
\left|\mathbf{P}\left\{\mu\left(x: A_{n-m, n} x=0\right)>r\right\}-\mathbf{P}\{\mu(n, m)>r\}\right| \leqslant \\
\leqslant \mathbf{P}\left\{\operatorname{rank}\left(A_{n-m, n}\right)<n-m\right\} .
\end{gathered}
$$

В свою очередь,

$$
\mathbf{P}\left\{\operatorname{rank}\left(A_{n-m, n}\right)<n-m\right\}=1-\prod_{k=0}^{n-m-1}\left(1-\frac{q^{k}}{q^{n}}\right) \leqslant \sum_{k=0}^{n-m-1} \frac{q^{k}}{2^{n}}<q^{-m} .
$$

Из (20) - (22) и леммы 1 следует, что для $\mathbf{P}\{\mu(n, m)>r\}$ выполнено неравенство (7). Теорема 1 доказана. 


\section{4. Доказательство следствия 1}

Для величин из правой части неравенства (7), очевидно, выполняются следующие соотношения:

1) при $n-m \rightarrow \infty, \lambda=O(1)$

$$
A\left(\lambda,\left|D_{r}^{*}\right|\right)=\lambda^{2} e^{q \lambda} \exp \left\{\frac{q^{m-n}}{\lambda}-1\right\}=O\left(\lambda q^{m-n}\right),
$$

2) при $\lambda=O(1)$

$$
B(\lambda)>\lambda^{2}\left(e-\frac{5}{2}\right)-\left(e^{\lambda}-1-\lambda-\frac{\lambda^{2}}{2}\right)=\lambda^{2}\left(e-\frac{5}{2}\right)+O\left(\lambda^{3}\right),
$$

3) при любых $n, m, r$, удовлетворяющих условиям $1 \leqslant m<n, 1 \leqslant r \leqslant n$, выполнено неравенство $\lambda_{r}(n, m) \geqslant q^{m-n} n$.

Равенство (10) вытекает из неравенства (7) и соотношений 1), 2), 3). Следствие 1 доказано.

\section{5. Доказательство теоремы 2}

Следуя [4], будем использовать обозначение

$$
D_{r}(d)=\left\{x \in K^{n}: 1 \leqslant \omega(x \oplus d) \leqslant r\right\}
$$

где $d \in K^{n}$, а символ $\oplus$ обозначает сложение векторов в $K^{n}$. Из определения функции $\rho\left(D_{r}\right)($ см. $(1)$ и $(2))$ и замкнутости множества $D_{r}$ относительно умножения векторов на ненулевые элементы поля следует, что

$$
\rho\left(D_{r}\right)=\max _{d \in K^{n}} \mathbf{P}\left\{\xi^{1} \oplus \xi^{2} \oplus d \in D_{r}\right\}=\max _{d \in K^{n}} \mathbf{P}\left\{\xi^{1} \oplus \xi^{2} \in D_{r}(d)\right\}
$$

где случайные векторы $\xi^{1}, \xi^{2}$ независимы и распределены равномерно на $D_{r}$ (подробнее см. раздел 5 в [4]). Известно, что при

$$
r \rightarrow \infty, \quad 0<r / n \leqslant c(q-1) / q, \quad 0<c<1,
$$

и любом $\varepsilon>0$ выполнены соотношения (см. лемму 7 в [4])

$$
\begin{gathered}
\mathbf{E} \omega\left(\xi^{1} \oplus \xi^{2}\right)=R(n, r)\left(1+O\left(r^{\varepsilon-1}\right)\right), \\
\mathbf{D} \omega\left(\xi^{1} \oplus \xi^{2}\right)=O\left(r^{1+\varepsilon}\right),
\end{gathered}
$$

где

$$
R(n, r)=r\left(2-\frac{r}{n} \frac{q}{q-1}\right) .
$$

Соотношение (24) вытекает из условия (11). Поэтому в рассматриваемом случае соотношения (25)) и (26)) выполнены.

Положим

$$
r_{1}=\left\lfloor R(n, r)-\frac{\beta r}{2}\right\rfloor, \quad r_{2}=\left\lfloor R(n, r)+\frac{\beta r}{2}\right\rfloor+1 .
$$


При выбранной величине $r_{1}$ получаем

$$
\begin{gathered}
\mathbf{P}\left\{\xi^{1} \oplus \xi^{2} \in D_{r}(d)\right\} \leqslant \\
\leqslant \mathbf{P}\left\{\left\{\omega\left(\xi^{1} \oplus \xi^{2}\right) \leqslant r_{1}\right\} \cup\left\{\omega\left(\xi^{1} \oplus \xi^{2}\right)>r_{2}\right\}\right\}+A_{1}(n, r),
\end{gathered}
$$

где

$$
A_{1}(n, r)=\sum_{u=\left\lfloor r_{1}\right\rfloor+1}^{r_{2}} \mathbf{P}\left\{\xi^{1} \oplus \xi^{2} \in D_{r}(d) \mid \omega\left(\xi^{1} \oplus \xi^{2}\right)=u\right\} \mathbf{P}\left\{\omega\left(\xi^{1} \oplus \xi^{2}\right)=u\right\}
$$

Так как распределения векторов $\xi^{1}, \xi^{2}$ и $\xi^{1} \oplus \xi^{2}$ равномерны на множествах

$$
D_{u}^{\prime}=\left\{x \in K^{n}: \omega(x)=u\right\},
$$

Tо

$$
\begin{aligned}
& \mathbf{P}\left\{\xi^{1} \oplus \xi^{2} \in D_{r}(d) \mid \omega\left(\xi^{1} \oplus \xi^{2}\right)=u\right\}= \\
& =\frac{\left|D_{r}(d) \cap\left\{\omega\left(\xi^{1} \oplus \xi^{2}=u\right)\right\}\right|}{\left|D_{u}^{\prime}\right|} \leqslant \frac{\left|D_{r}(d)\right|}{\left|D_{u}^{\prime}\right|}=\frac{\left|D_{r}\right|}{(q-1)^{u} C_{n}^{u}} .
\end{aligned}
$$

Следовательно,

$$
\begin{aligned}
A_{1}(n, r) & \leqslant \frac{\left|D_{r}\right|}{(q-1)^{u} C_{n}^{u}} \leqslant \frac{\left|D_{r}\right|}{(q-1)^{r_{1}}} \max _{r_{1}+1 \leqslant u \leqslant r_{2}} \frac{1}{C_{n}^{u}}= \\
& =\frac{\left|D_{r}\right|}{(q-1)^{r_{1}}} \max \left\{\frac{1}{C_{n}^{r_{1}}}, \frac{1}{C_{n}^{n-r_{2}}}\right\} .
\end{aligned}
$$

Так как

$$
\begin{gathered}
r_{1}=R(n, r)-\frac{\beta r}{2}+\theta_{1}=2 R(n, r)-\left(R(n, r)+\frac{\beta r}{2}\right)+\theta_{1}= \\
=2 R(n, r)-n+n-r_{2}+\theta_{2}
\end{gathered}
$$

при некоторых $\theta_{1}, \theta_{2},\left|\theta_{1}\right| \leqslant 1,\left|\theta_{2}\right| \leqslant 2$, то

$$
2 R(n, r)-n=2 r\left(2-\frac{r}{n} \frac{q}{q-1}\right)-n=n\left(\frac{4 r}{n}-\left(\frac{r}{n}\right)^{2} \frac{2 q}{q-1}-1\right) .
$$

Заметим, что многочлен

$$
4 x-x^{2} \frac{2 q}{q-1}-1
$$

при

$$
0<x<\frac{q-1}{q}\left(1-\sqrt{1-\frac{q}{2(q-1)}}\right)
$$

принимает только отрицательные значения. Поэтому из (11) следует, что $2 R(n, r)-$ $n<0$, а $r_{1}<n-r_{2}+2$, а из $(30)-$ что

$$
A_{1}(n, r) \leqslant \frac{\left|D_{r}\right|}{(q-1)^{r_{1}}} \frac{1}{C_{n}^{r_{1}-2}}<\frac{r(q-1)^{r}}{(q-1)^{r_{1}}} \frac{C_{n}^{r}}{C_{n}^{r_{1}-2}} .
$$

Отметим, что при любых натуральных $n>m>k$

$$
C_{n}^{k} \leqslant\left(\frac{m}{n-m}\right)^{m-k} C_{n}^{m}
$$


а в силу (27) и (11)

$$
\begin{gathered}
r_{1}-r>R(n, r)-\frac{\beta r}{2}-r=r\left(1-\frac{r}{n} \frac{q}{q-1}-\frac{\beta}{2}\right)> \\
>r\left(1-(1-\beta)\left(1-\sqrt{1-\frac{q}{2(q-1)}}\right)-\frac{\beta}{2}\right)= \\
=r\left((1-\beta) \sqrt{1-\frac{q}{2(q-1)}}+\frac{\beta}{2}\right)>\beta^{\prime} r,
\end{gathered}
$$

где $\beta^{\prime}>0$. Поэтому из (31) следует равенство

$$
A_{1}(n, r)=O\left(e^{-\gamma r}\right) \quad \text { при некотором } \gamma>0 .
$$

Теперь оценим $\mathbf{P}\left\{\left\{\omega\left(\xi^{1} \oplus \xi^{2}\right) \leqslant r_{1}\right\} \cup\left\{\omega\left(\xi^{1} \oplus \xi^{2}\right)>r_{2}\right\}\right\}$. Имеем:

$$
\begin{gathered}
\left\{\omega\left(\xi^{1} \oplus \xi^{2}\right) \leqslant r_{1}\right\}=\left\{\omega\left(\xi^{1} \oplus \xi^{2}\right)-\mathbf{E} \omega\left(\xi^{1} \oplus \xi^{2}\right) \leqslant r_{1}-\mathbf{E} \omega\left(\xi^{1} \oplus \xi^{2}\right)\right\}= \\
=\left\{\mathbf{E} \omega\left(\xi^{1} \oplus \xi^{2}\right)-\omega\left(\xi^{1} \oplus \xi^{2}\right) \geqslant \mathbf{E} \omega\left(\xi^{1} \oplus \xi^{2}\right)-r_{1}\right\}
\end{gathered}
$$

и

$$
\left\{\omega\left(\xi^{1} \oplus \xi^{2}\right)>r_{2}\right\}=\left\{\omega\left(\xi^{1} \oplus \xi^{2}\right)-\mathbf{E} \omega\left(\xi^{1} \oplus \xi^{2}\right)>r_{2}-\mathbf{E} \omega\left(\xi^{1} \oplus \xi^{2}\right)\right\} .
$$

Заметим, что разности $\mathbf{E} \omega\left(\xi^{1} \oplus \xi^{2}\right)-r_{1}$ и $r_{2}-\mathbf{E} \omega\left(\xi^{1} \oplus \xi^{2}\right)$ в этих формулах положительны (начиная с некоторого момента). Значит,

$$
\begin{gathered}
\left.\mathbf{P}\left\{\left\{\omega\left(\xi^{1} \oplus \xi^{2}\right) \leqslant r_{1}\right\} \cup\left\{\omega\left(\xi^{1} \oplus \xi^{2}\right)>r_{2}\right\}\right\}\right) \leqslant \\
\leqslant \mathbf{P}\left\{\left|\mathbf{E} \omega\left(\xi^{1} \oplus \xi^{2}\right)-\omega\left(\xi^{1} \oplus \xi^{2}\right)\right| \geqslant E\right\},
\end{gathered}
$$

где

$$
E=\min \left\{\mathbf{E} \omega\left(\xi^{1} \oplus \xi^{2}\right)-r_{1}, r_{2}-\mathbf{E} \omega\left(\xi^{1} \oplus \xi^{2}\right)\right\} .
$$

Далее воспользуемся соотношениями (25) - (28):

$$
\begin{aligned}
\mathbf{E} \omega\left(\xi^{1} \oplus \xi^{2}\right)-r_{1}= & R(n, r)\left(1+O\left(r^{\varepsilon-1}\right)\right)-R(n, r)+\frac{\beta r}{2}= \\
& =\frac{\beta r}{2}\left(1+O\left(r^{\varepsilon-1}\right)\right), \\
r_{2}-\mathbf{E} \omega\left(\xi^{1} \oplus \xi^{2}\right)= & R(n, r)+\frac{\beta r}{2}-R(n, r)\left(1+O\left(r^{\varepsilon-1}\right)\right)= \\
& =\frac{\beta r}{2}\left(1+O\left(r^{\varepsilon-1}\right)\right) .
\end{aligned}
$$

Поэтому

$$
E=\frac{\beta r}{2}\left(1+O\left(r^{\varepsilon-1}\right)\right)
$$

и, применяя к правой части (33) неравенство Чебышёва, получаем:

$$
\begin{aligned}
\mathbf{P}\left\{\left\{\omega\left(\xi^{1} \oplus \xi^{2}\right)\right.\right. & \left.\left.\leqslant r_{1}\right\} \cup\left\{\omega\left(\xi^{1} \oplus \xi^{2}\right)>r_{2}\right\}\right\} \leqslant \frac{1}{E^{2}} \mathbf{D} \omega\left(\xi^{1} \oplus \xi^{2}\right)= \\
& =\frac{O\left(r^{1+\varepsilon}\right)}{\left(\frac{\beta r}{2}\left(1+O\left(r^{\varepsilon-1}\right)\right)\right)^{2}}=O\left(r^{\varepsilon-1}\right) .
\end{aligned}
$$

Теперь из (23), (29), (32) и (33) следует (12). Теорема 2 доказана. 


\section{6. Доказательство теоремы 3}

Нам понадобится несколько вспомогательных утверждений.

Лемма 2. Пусть $1<r \leqslant n(q-1) /(q+e-1)$. Тогда

$$
Q(n, r) \cdot H(n, r) \leqslant \sum_{k=1}^{r} C_{n}^{k}(q-1)^{k} \leqslant Q(n, r)
$$

¿əe

$$
\begin{gathered}
Q(n, r)=C_{n}^{r}(q-1)^{r} \frac{(q-1)(n-r)}{(q-1)(n-r)-r}, \\
H(n, r)=\left(1+\frac{\lfloor\ln r\rfloor}{n-r}\right)\left(1+\frac{q \cdot\lfloor\ln r\rfloor}{(q-1)(n-r)-r}\right)^{-1}\left(1-\frac{1}{r}\right) .
\end{gathered}
$$

Доказательство леммы 2. Нетрудно показать, что

$$
\begin{gathered}
\sum_{k=1}^{r} C_{n}^{k}(q-1)^{k}=C_{n}^{r}\left((q-1)^{r}+\sum_{k=1}^{r-1}(q-1)^{k} \prod_{i=0}^{r-k-1} \frac{C_{n}^{r-i-1}}{C_{n}^{r-i}}\right) \\
=C_{n}^{r}\left((q-1)^{r}+\sum_{k=1}^{r-1}(q-1)^{k} \prod_{i=0}^{r-k-1} \frac{r-i}{n-r+i+1}\right) .
\end{gathered}
$$

Так как

$$
\frac{r-i}{n-r+i+1} \leqslant \frac{r}{n-r}, \quad 0 \leqslant i \leqslant r<n,
$$

то из (38) следует, что

$$
\begin{gathered}
\sum_{k=1}^{r} C_{n}^{k}(q-1)^{k} \leqslant C_{n}^{r} \sum_{k=1}^{r}(q-1)^{k}\left(\frac{r}{n-r}\right)^{r-k}= \\
=C_{n}^{r}(q-1)^{r} \sum_{k=1}^{r} \frac{1}{(q-1)^{r-k}}\left(\frac{r}{n-r}\right)^{r-k}=C_{n}^{r}(q-1)^{r} \frac{(q-1)(n-r)}{(q-1)(n-r)-r},
\end{gathered}
$$

и верхняя оценка в (35) доказана.

Чтобы получить нижнюю оценку, воспользуемся вытекающими из (38) и (39) неравенствами

$$
\begin{gathered}
\sum_{k=1}^{r} C_{n}^{k}(q-1)^{k} \geqslant C_{n}^{r}\left((q-1)^{r}+\sum_{k=r-\lfloor\ln r\rfloor}^{r-1}(q-1)^{k} \prod_{i=0}^{r-k-1} \frac{r-i}{n-r+i+1}\right) \geqslant \\
\geqslant C_{n}^{r}\left(\sum_{k=r-\lfloor\ln r\rfloor}^{r}(q-1)^{k}\left(\frac{r-\lfloor\ln r\rfloor}{n-r+\lfloor\ln r\rfloor}\right)^{r-k}\right)= \\
=C_{n}^{r}(q-1)^{r}\left(\sum_{k=r-\lfloor\ln r\rfloor}^{r} \frac{1}{(q-1)^{r-k}}\left(\frac{r-\lfloor\ln r\rfloor}{n-r+\lfloor\ln r\rfloor}\right)^{r-k}\right)= \\
=C_{n}^{r}(q-1)^{r} \frac{(q-1)(n-r+\lfloor\ln r\rfloor)}{(q-1)(n-r+\lfloor\ln r\rfloor)-r+\lfloor\ln r\rfloor} \times
\end{gathered}
$$




$$
\times\left(1-\left(\frac{r-\lfloor\ln r\rfloor}{(q-1)(n-r+\lfloor\ln r\rfloor)}\right)^{\lfloor\ln r\rfloor+1}\right) .
$$

Так как $r \leqslant n(q-1) /(q+e-1)$, то

$$
\frac{r-\lfloor\ln r\rfloor}{(q-1)(n-r+\lfloor\ln r\rfloor)}<e^{-1},
$$

и приходим к нижней оценке в (35). Лемма 2 доказана.

Из леммы 2 сразу вытекает следствие 3.

Следствие 3. Пусть $r \rightarrow \infty$ maк, что $r \leqslant n(q-1) /(q+e-1)$. Тогда

$$
\sum_{k=1}^{r} C_{n}^{k}(q-1)^{k}=C_{n}^{r}(q-1)^{r} \frac{(q-1)(n-r)}{(q-1)(n-r)-r}\left(1+O\left(\frac{\ln r}{r}\right)\right) .
$$

Лемма 3. Пусть $n \rightarrow \infty, s^{2}=o(n), \kappa=\kappa(n, m)$ является решением уравнения (15) и выполнено условие (17). Тогда

$$
\begin{gathered}
(q-1)^{\lfloor n \kappa\rfloor+s} C_{n}^{\lfloor n \kappa\rfloor+s}= \\
=q^{n-m}\left(\frac{1-\kappa}{\kappa}(q-1)\right)^{s-\{n \kappa\}}\left(1+O\left(\frac{s^{2}+1}{n}\right)\right) .
\end{gathered}
$$

Доказательство леммы 3. Положим

$$
r=\lfloor\kappa n\rfloor+s=n \kappa+L(n), \quad \text { где } \quad L(n)=s-\{n k\}=O(|s|+1) .
$$

Обозначим

$$
u=1-\frac{1}{q}, \quad H(x)=x \ln \frac{x}{u}+(1-x) \ln \frac{1-x}{1-u}, \quad w=\frac{r}{n}=\kappa+\frac{L(n)}{n} .
$$

Тогда

$$
\begin{gathered}
H(w)=(w \ln w+(1-w) \ln (1-w))-(w \ln u+(1-w) \ln (1-u))= \\
=\left(\kappa \ln \kappa+(1-\kappa) \ln (1-\kappa)+\frac{L(n)}{n} \ln \frac{\kappa}{1-\kappa}\right)- \\
-\left(\kappa \ln \frac{u}{1-u}+\ln (1-u)+\frac{L(n)}{n} \ln \frac{u}{1-u}\right)+O\left(\frac{L^{2}(n)+1}{n^{2}}\right)= \\
=\kappa \ln \kappa+(1-\kappa) \ln (1-\kappa)-\frac{L(n)}{n} \ln \left(\frac{1-\kappa}{\kappa}(q-1)\right)- \\
-\kappa \ln (q-1)+\ln q+O\left(\frac{L^{2}(n)+1}{n^{2}}\right)= \\
=\frac{m}{n} \ln q-\frac{1}{n} \ln \sqrt{2 \pi n \kappa(1-\kappa)}-\frac{L(n)}{n} \ln \left(\frac{1-\kappa}{\kappa}(q-1)\right)+O\left(\frac{L^{2}(n)+1}{n^{2}}\right) .
\end{gathered}
$$

Здесь мы воспользовались равенством (15).

Согласно теореме 4 из главы 5 в [8],

$$
(q-1)^{r} C_{n}^{r}=q^{n} \cdot C_{n}^{r} u^{r}(1-u)^{n-r}=
$$




$$
=q^{n} \cdot \frac{1}{\sqrt{2 \pi n w(1-w)}} \exp \{-n H(w)+\theta(r, n)\},
$$

где

$$
|\theta(r, n)|<\frac{1}{12 n w(1-w)}=O\left(\frac{1}{n}\right)
$$

Вместе с этим

$$
\frac{1}{\sqrt{2 \pi n w(1-w)}}=\exp \left\{-\ln \sqrt{2 \pi n \kappa(1-\kappa)}+O\left(\frac{|L(n)|+1}{n}\right)\right\} .
$$

Из $(42)-(44)$ и равенства $L(n)=s-\{n k\}=O(|s|+1)$ получаем

$$
\begin{gathered}
(q-1)^{n \kappa+L(n)} C_{n}^{n \kappa+L(n)}= \\
=q^{n-m}\left(\frac{1-\kappa}{\kappa}(q-1)\right)^{L(n)}\left(1+O\left(\frac{s^{2}+1}{n}\right)\right) .
\end{gathered}
$$

Из (45) следует (41). Лемма 3 доказана.

Лемма 4. Пусть $n \rightarrow \infty, s^{2}=o(n), \kappa=\kappa(n, m)$ является решением уравнения (15) и выполнено условие (17). Тогда

$$
\lambda_{\lfloor n \kappa\rfloor+s}(n, m)=q_{s}(n, m)\left(1+O\left(\frac{s^{2}+\ln n}{n}\right)\right),
$$

где $q_{s}(n, m)$ определяется по формуле (16).

Доказательство леммы 4. Согласно определениям и лемме 2

$$
\begin{gathered}
\lambda_{r}(n, m)=\lambda_{\lfloor n \kappa\rfloor+s}(n, m)=\frac{\left|D_{\lfloor n \kappa\rfloor+s}^{*}\right|}{q^{n-m}}=\frac{1}{q^{n-m}(q-1)} \sum_{i=1}^{\lfloor n \kappa\rfloor+s} C_{n}^{i}(q-1)^{i}= \\
=\frac{1}{q^{n-m}(q-1)} C_{n}^{r}(q-1)^{r} \frac{(q-1)(n-r)}{(q-1)(n-r)-r}\left(1+O\left(\frac{\ln n}{n}\right)\right),
\end{gathered}
$$

где $Q(n,\lfloor n \kappa\rfloor+s)$ - величина, определяемая согласно формуле (36), а множество $D_{r}^{*}$ было определено перед формулой (4). Из (41) и соотношения

$$
\begin{gathered}
\frac{(q-1)(n-r)}{(q-1)(n-r)-r}=\frac{(q-1)(n-\lfloor n \kappa\rfloor-s)}{(q-1)(n-\lfloor n \kappa\rfloor-s)-\lfloor n \kappa\rfloor-s}= \\
=\frac{(q-1)(1-\kappa)}{(q-1)(1-\kappa)-\kappa}\left(1+O\left(\frac{|s|+1}{n}\right)\right),
\end{gathered}
$$

следует (46). Лемма 4 доказана.

Теперь утверждения (18), (19) теоремы 3 следуют из оценок (10), (12) и (46). Теорема 3 доказана. 


\section{Список литературы}

1. Питерсон У., Уэлдон Э., Кодъ, исправляющие ошибки, М.: Мир, 1976.

2. Мак-Вильямс Ф. Дж., Слоэн Н. Дж. А., Теория кодов, исправляющих ошибки, М.: Связь, 1979.

3. Сидельников В. М., Теория кодирования. Справочник по приниипам и методам кодирования, М.: МГУ, 2006.

4. Копытцев В. А., Михайлов В. Г., "Теоремы пуассоновского типа для числа специальных решений случайного линейного включения", Дискретная математика, 22:2 (2010), $3-21$.

5. Копытцев В. А., Михайлов В. Г., "Явные оценки точности пуассоновской аппроксимации для распределения числа решений случайных включений", Математические вопросы криптографии, 6:1 (2015), 57-79.

6. Зубков А. М., Круглов В. И., "Моментные характеристики весов векторов в случайных двоичных кодах", Математические вопросы криптографии, 3:4 (2012), 55-70.

7. Зубков А. М., Круглов В. И., "Статистические характеристики весовых спектров случайных линейных кодов над $G F(p)$ ", Математические вопросы криптографии, 5:1 (2014), 27-38.

8. Боровков А. А., Теория вероятностей, Наука, Москва, 1976.

Статья поступила 11.03.2015. 